\title{
Responsabilité personnelle
}

\section{Anna Sax}

Lic. oec. publ., MHA, membre de la rédaction

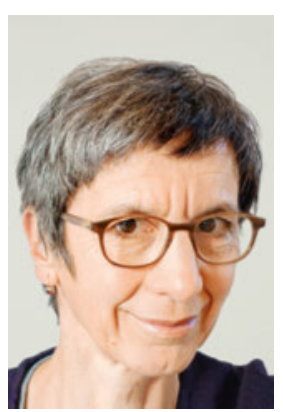

Tous les canaux d'information évoquent, une fois de plus, la hausse des coûts dans le secteur de la santé et bien entendu, tous les experts et politiciennes de la santé ont un remède à proposer: moins de prestations, d'hôpitaux, de liberté dans le choix du médecin, des franchises plus élevées, ainsi que davantage de concurrence et, surtout, de responsabilité personnelle. Plus de responsabilité personnelle signifie payer plus de sa poche, car c'est bien connu, l'être humain examine d'abord son portemonnaie avant de tomber malade. En tant qu'économiste, je sais en outre que moralement, c'est un risque-tout. Autrement dit, une fois sa prime d'assurance payée, il perd toutes ses inhibitions et veut tout. Tout de suite. Daniel Habegger de santésuisse a par exemple déclaré à $N Z Z$ que parce que les primes augmentaient constamment, les assurés se sentaient confortés dans leur droit de courir chez le médecin à la moindre bagatelle [1]. Cette course fait flamber les coûts, donc les primes, donc le nombre de consultations. Un cercle vicieux que nous ne pourrons briser qu'en augmentant la responsabilité personnelle.

«Responsabilité personnelle» a l'étoffe d'un mot de l'année, ou même de la décennie! C'est une formidable qualité, un multitalent! Grâce à elle, nous nous informons, nous savons nous situer sur le marché, nous prenons conscience de ce que nous voulons, de ce dont nous avons vraiment besoin et de la somme que nous acceptons de débourser pour cela. Grâce à elle, nous prenons le temps de réfléchir avant de nous mettre à courir. Elle nous rend meilleurs.

Autre bon point: il est facile de distinguer les gens responsables de ceux qui ne le sont pas. Les irresponsables sont ceux qui, à la fin de l'année, une fois la franchise atteinte, se rendent spécialement chez le médecin, juste parce que c'est la caisse-maladie qui paie. Et ce n'est pas tout: les irresponsables sont obèses, fumeurs et passent leur temps avachis devant un écran au lieu de faire du sport. Ils mangent trop de sucre, trop de graisses, pas assez de légumes. Ils se moquent des recommandations des spécialistes et, quand ils se sentent malades, ils courent chez le médecin sans réfléchir. Et nous, les non-fumeuses minces, toniques, sportives, nous payons pour eux. Nous qui assumons notre responsabilité personnelle, nous sommes punies par la hausse sans fin des primes de caisse-maladie. Alors il ne faut pas s'étonner si, comme Daniel Oertle l'a récemment très bien expliqué dans ce journal [2], nous nous permettons une petite IRM vite faite à la clinique du sport.

Attention, on passe maintenant aux choses concrètes: qui exactement n'assume pas sa responsabilité personnelle et doit donc payer plus? Tous ceux dont l'IMC est supérieur ou égal à 25 ? Ou à 30 ? Faut-il tenir un registre des habitudes alimentaires? Les sanctions débutentelles à cinq, dix ou 40 cigarettes par jour? De combien doit être la franchise minimale afin d'éviter d'une part que les gens courent chez les médecins pour des broutilles et d'autre part, qu'ils n'attendent pas jusqu'à ce qu'il soit trop tard pour consulter? Selon le dernier rapport du Commonwealth [3], en Suisse, la part d'adultes qui ont renoncé à des services médicaux pour des raisons financières est passée de 10,3 à 22,5 pour cent entre 2010 et 2016. La part que l'assuré paie de sa poche, c'est-à-dire les dépenses de santé qui ne sont couvertes par aucune assurance, est plus élevée en Suisse que partout ailleurs en Europe. Et ceux qui comme santésuisse et la majorité du Parlement réclament une hausse des franchises afin d'encourager la responsabilité personnelle ne devraient pas oublier que les primes et la participation aux coûts ont plus que doublé depuis 1996, alors que les salaires n'ont augmenté que de 23 pour cent. Depuis deux décennies, nous transférons la charge du financement de la santé vers les personnes socialement défavorisées et les malades chroniques. Ce faisant, nous n'encourageons pas notre responsabilité personnelle, nous refusons d'assumer notre responsabilité vis-à-vis de la communauté.

\section{Références}

1 NZZ du 8 décembre 2016

2 Oertle D. Die Hausärzte sterben aus: Na und? (Les médecins de famille sont en voie de disparition. Et alors?) Bull med suisses. 2016;97(51-52):1804-5.

3 International Health Policy Survey 2016 du Commonwealth Fund. 